\title{
Mediterranean Fruit Fly, Ceratitis capitata (Wiedemann) (Insecta: Diptera: Tephritidae) ${ }^{1}$
}

\author{
M. C. Thomas, J. B. Heppner, R. E. Woodruff, H. V. Weems, G. J. Steck and T. R. Fasulo²
}

\section{Introduction}

The Mediterranean fruit fly, Ceratitis capitata (Wiedemann), is one of the world's most destructive fruit pests. The species originated in sub-Saharan Africa and is not known to be established in the United States. When it has been detected in Florida and California, especially in recent years, each infestation necessitated intensive and massive eradication and detection procedures so that the pest did not become established.

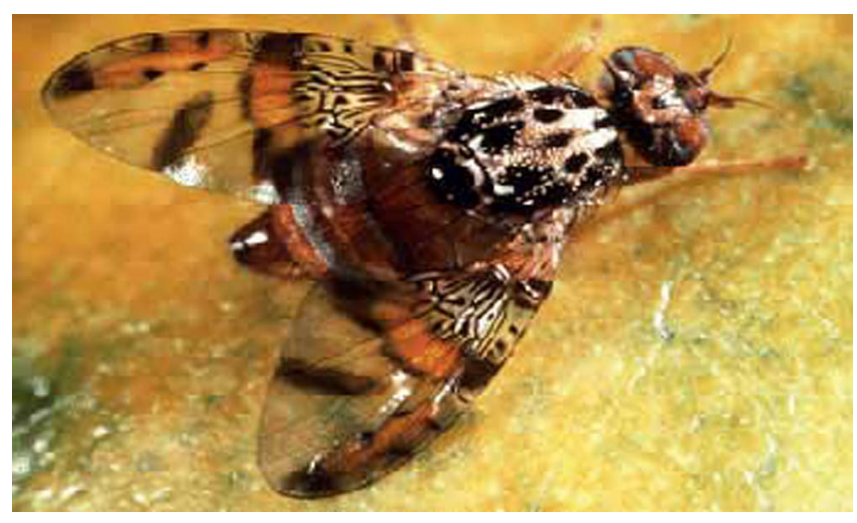

Figure 1. Adult male Mediterranean fruit fly, Ceratitis capitata (Wiedemann). Credits: USDA
Because of its wide distribution over the world, its ability to tolerate cooler climates better than most other species of tropical fruit flies, and its wide range of hosts, it is ranked first among economically important fruit fly species. Its larvae feed and develop on many deciduous, subtropical, and tropical fruits and some vegetables. Although it may be a major pest of citrus, often it is a more serious pest of some deciduous fruits, such as peach, pear, and apple. The larvae feed upon the pulp of host fruits, sometimes tunneling through it and eventually reducing the whole to a juicy inedible mass. In some of the Mediterranean countries, only the earler varieties of citrus are grown, because the flies develop so rapidly that late season fruits are too heavily infested to be marketable. Some areas have had almost $100 \%$ infestation in stone fruits. Harvesting before complete maturity also is practiced in Mediterranean areas generally infested with this fruit fly. In this age of jet transportation, the "medfly" can be transported from one part of the world to some distant place in a matter of hours, which greatly complicates efforts to contain it within its present distribution. Once it is established, eradication efforts may be extremely

1. This document is EENY-214 (originally published as DPI Entomology Circulars 4, 230 and 273), one of a series of Featured Creatures from the Entomology and Nematology Department, Florida Cooperative Extension Service, Institute of Food and Agricultural Sciences, University of Florida. Published: July 2001. Revised: September 2001. This document is also available on Featured Creatures Website at http://creatures.ifas.ufl.edu. Please visit the EDIS Website at http://edis.ifas.ufl.edu.

2. M. C. Thomas, J. B. Heppner, R. E. Woodruff, H. V. Weems, G. J. Steck, Florida Department of Agriculture and Consumer Services, Division of Plant Industry; and T. R. Fasulo, Entomology and Nematology Department, University of Florida, Gainesville, FL.

The Institute of Food and Agricultural Sciences (IFAS) is an Equal Employment Opportunity - Affirmative Action Employer authorized to provide research, educational information and other services only to individuals and institutions that function without regard to race, creed, color, religion, age, disability, sex, sexual orientation, marital status, national origin, political opinions or affiliations. For information on obtaining other extension publications, contact your county Cooperative Extension Service office. Florida Cooperative Extension Service / Institute of Food and Agricultural Sciences / University of Florida / Larry R. Arrington, Interim Dean 
difficult and expensive. In addition to reduction of crop yield, infested areas have the additional expense of control measures and costly sorting processes for both fresh and processed fruit and vegetables. Some countries maintain quarantines against the medfly, which could jeopardize some fresh fruit markets if it should become established in Florida.

\section{Synonyms}

\section{Ceratitis citriperda MacLeay}

Ceratitis hispanica De Brême

Paradalaspis asparagi Bezzi

Tephritis capitata Wiedemann

Ceratitis capitata (Wiedemann)

\section{Distribution}

Mediterranean fruit fly infestations in the United States have occurred in Hawaii since 1910; in Florida from April 1929 to July 1930, April 1956 to

November 1957, June 1962 to February 1963, June to August 1963, 3-14 August 1981, and April to August 1998, with one or two flies found in various counties during 1967, 1983 to 1988, 1990 to 1991 and in May to October, 1997; in Texas from June to July 1966; and in California in 1975, and chronically after 1980.

Other infested countries are $(*=$ countries with recorded infestations): Albania, Algeria, Angola, Argentina, Australia, Austria*, Azores, Balearic Islands, Belgium*, Bolivia, Botswana, Brazil, Burkina Faso, Burundi, Cameroon, Canary Islands, Cape Verde Islands*, Colombia, Costa Rica, Crete, Cyprus, Dahomey, Ecuador, Egypt, El Salvador, Ethiopia, France, Germany*, Ghana, Greece, Guatemala, Guinea, Honduras, Hungary*, Israel, Italy, Ivory Coast, Jordan, Kenya, Lebanon, Liberia, Libya, Madagascar, Madeira Islands, Malagasy Republic, Malawi, Mali, Malta, Mauritius*, Mexico (chronic) (near Guatemalan border), Morocco, Mozambique, Netherlands*, Nicaragua, Niger, Nigeria, Panama, Paraguay, Peru, Portugal, Reunion, Rhodesia, Rwanda, Saint Helena, San Miguel (Azores), Sardinia, Saudi Arabia, Senegal, Seychelles, Sicily, Sierra Leone, South Africa, Southern Rhodesia, Spain, Sudan, Switzerland*,
Syria, Tanzania, Tasmania, Togo, Tunisia, Turkey, Uganda, Uruguay, Venezuela, Yugoslavia, Zaire, and Zambia.

New Zealand experienced a small incursion of this pest in Auckland during 1996. The Medfly was eradicated and there have been no further detections (Stephenson 2001).

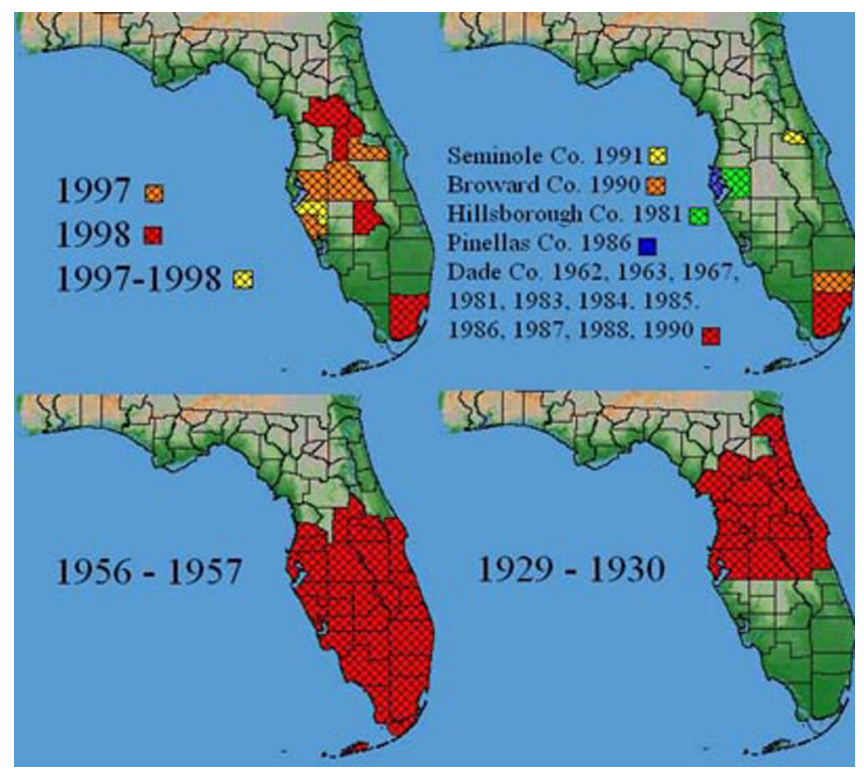

Figure 2. Incidence of the Mediterranean fruit fly, Ceratitis capitata (Wiedemann), in Florida. Credits: G. J. Steck and B. D. Sutton, Division of Plant Industry

\section{Identification}

The medfly has no near relatives in the Western Hemisphere. The adults are slightly smaller than a house fly and have picture wings typical of fruit flies. They can be distinguished fairly readily from any of the native fruit flies of the New World.

Egg: very slender, curved, $1 \mathrm{~mm}$ long, smooth and shiny white. Micropylar region distinctly tubercular.

Larva: Larva are white with a typical fruit fly larval shape (cylindrical maggot-shape, elongate, anterior end narrowed and somewhat recurved ventrally, with anterior mouth hooks, and flattened caudal end); last instar usually 7 to $9 \mathrm{~mm}$ in length, with 8 ventral fusiform areas; anterior buccal carinae usually 9 to 10 in number; anterior spiracles usually nearly straight on dorsal edge of tubule row (often more straight than illustrated); usually with 9 to 10 tubules (may be 7 to 11 ). 


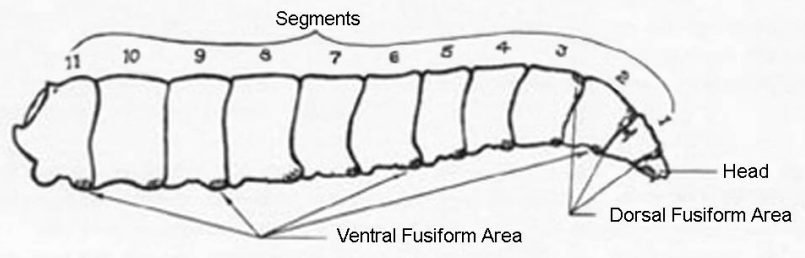

Figure 3. Lateral view of a mature larva of the Mediterranean fruit fly, Ceratitis capitata (Wiedemann). Credits: Division of Plant Industry

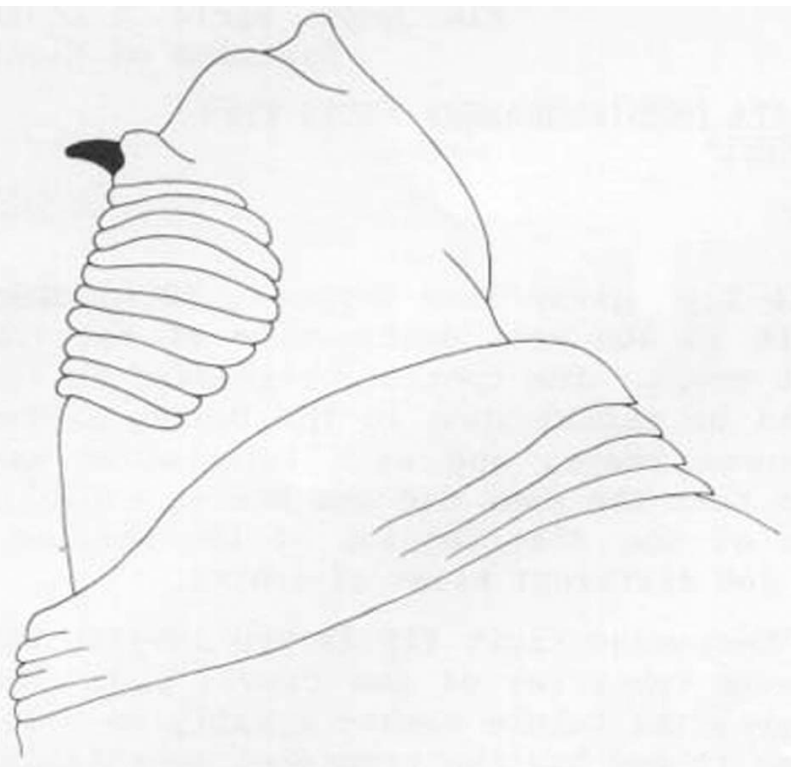

Figure 4. Head and buccal carinae of larva of the Mediterranean fruit fly, Ceratitis capitata (Wiedemann). Credits: Division of Plant Industry

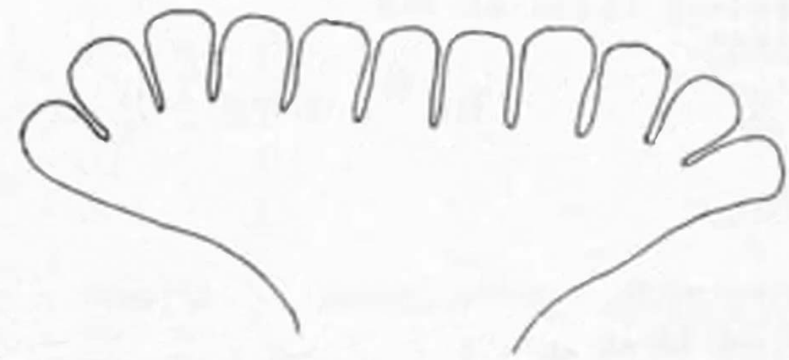

Figure 5. Anterior spiracles of larva. Credits: Division of Plant Industry

Cephalo-pharyngeal skeleton with large convex mouth hook each side, approximately $2 \mathrm{X}$ hypostome length; hypostomium with prominent, rounded subhypostomium; post-hypostomial plates curved dorsally to dorsal bridge, fused with sclerotized rays of central area of dorsal wing plate; parastomium prominent; anterior of dorsal bridge with a prominent sclerotized point; dorsal wing plate nearly as long as pharyngeal plate; median area relatively unsclerotized; pharyngeal plate elongate, with prominent median hood and anterior sclerotized area.

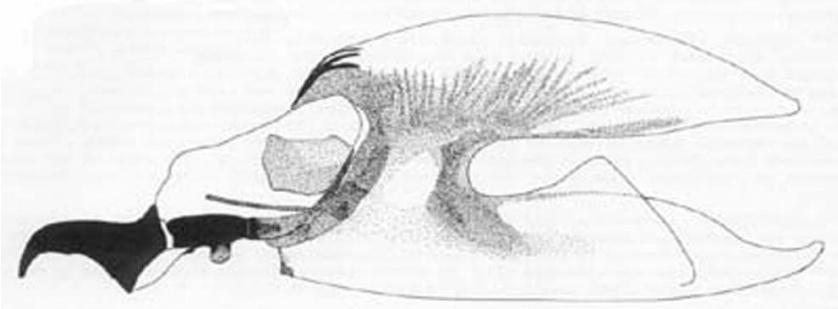

Figure 6. Larval cephalo-pharyngeal skeleton of the Mediterranean fruit fly, Ceratitis capitata (Wiedemann). Credits: Division of Plant Industry

Caudal end with bifurcate or paired dorsal papillules (D1 and D2) on small mount of relatively flat plate; intermediate papillules (I1-2) as a line of fused elevations on a very enlarged subspiracular tubercle, plus a remote $\mathrm{I} 3$ at approximately 45 degrees from I1-2; L1 on the median edge of the caudal end; V1 not prominent; posterior spiracles elongate ( 4.5 to $5 \mathrm{X}$ width), with dorsal and ventral spiracles angled away from relatively planar median spiracle; interspiracular processes (hairs) usually not branched; anal lobe bifid or entire.

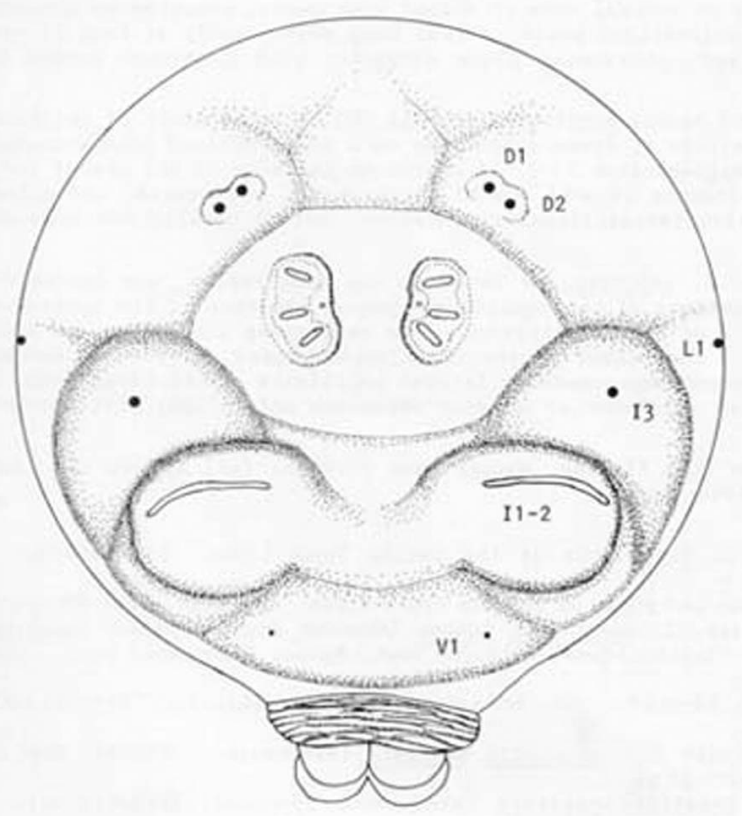

Figure 7. Caudal end of larva of the Mediterranean fruit fly, Ceratitis capitata (Wiedemann). Credits: Division of Plant Industry 


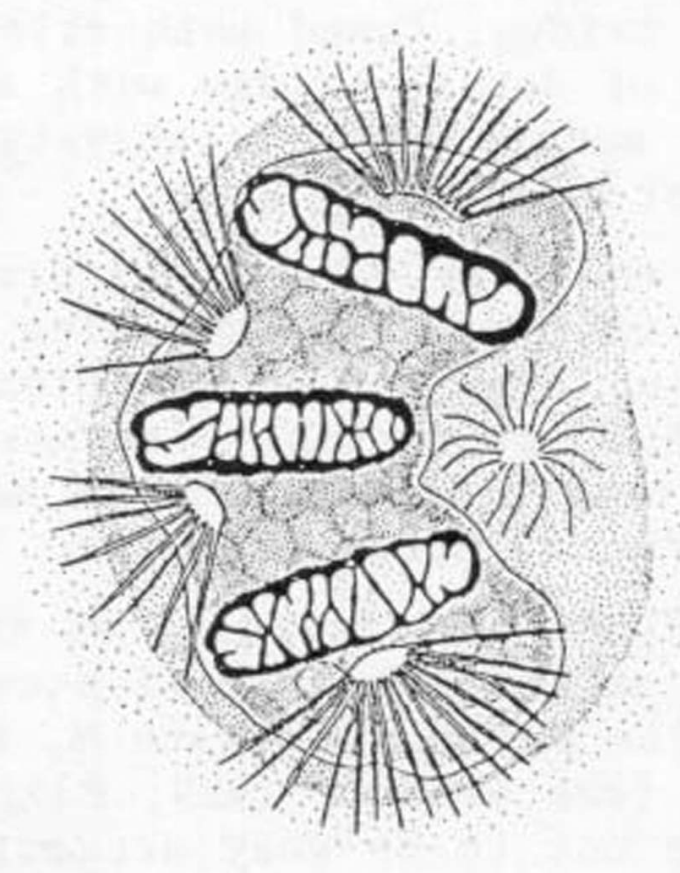

Figure 8. Posterior spriacles (left side) (after Phillips 1946) of a larva of the Mediterranean fruit fly, Ceratitis capitata (Wiedemann). Credits: Division of Plant Industry

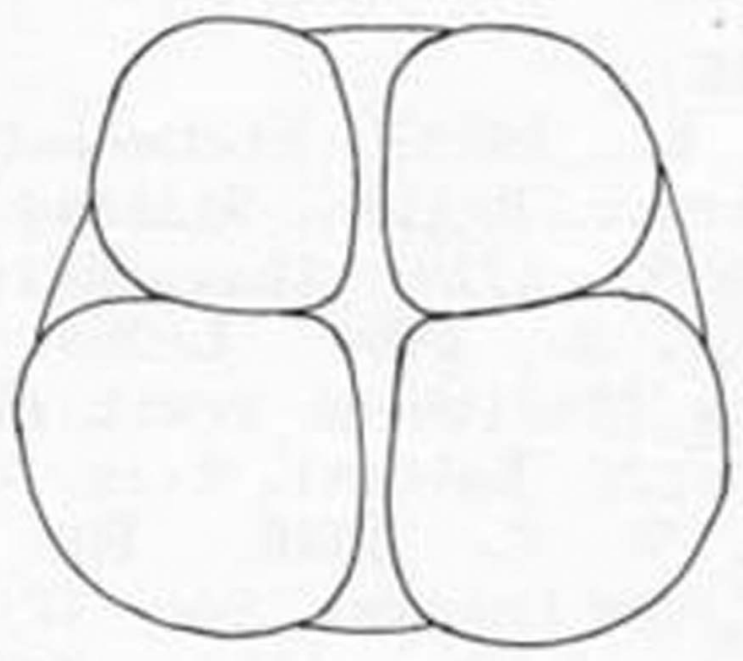

Figure 9. Anal lobes of larva. Credits: Division of Plant Industry

Pupa: cylindrical, 4 to $4.3 \mathrm{~mm}$ long, dark reddish brown, resembling swollen grain of wheat.

Adult: length 3.5 to $5 \mathrm{~mm}$. Yellowish with brown tinge, especially on abdomen, legs, and some markings on wings. Lower corners of face with white setae. Eyes reddish purple (fluoresce green, turning
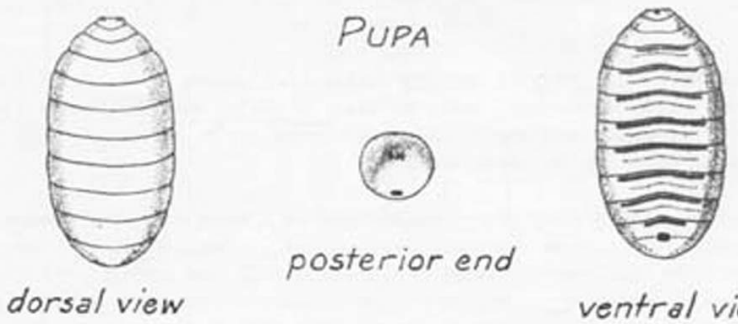

dorsal view

ventral view

Figure 10. Pupal view. Credits: Division of Plant Industry

blackish within 24 hours after death). Ocellar bristles present. Male has pair of bristles with enlarged spatulate tips next to inner margins of eyes. Thorax creamy white to yellow with characteristic pattern of black blotches. Light areas with very fine white bristles. Humeral bristles present. Dorsocentral bristles anterior of halfway point between supraalar and acrostichal bristles. Scutellum inflated and shiny black. Abdomen oval with fine black bristles scattered on dorsal surface and two narrow transverse light bands on basal half. Extended ovipositor $1.2 \mathrm{~mm}$ long. Wings, usually held in a drooping position on live flies, are broad and hyaline with black, brown, and brownish yellow markings. Wide brownish yellow band across middle of wing. Apex of anal cell elongate. Dark streaks and spots in middle of cells in and anterior to anal cell.

The males are easily separated from all other members of this family by the black pointed expansion at the apex of the anterior pair of orbital setae. The females can be separated from most other species by the characteristic yellow wing pattern and the apical half of the scutellum being entirely black (White and Elson-Harris 1994).

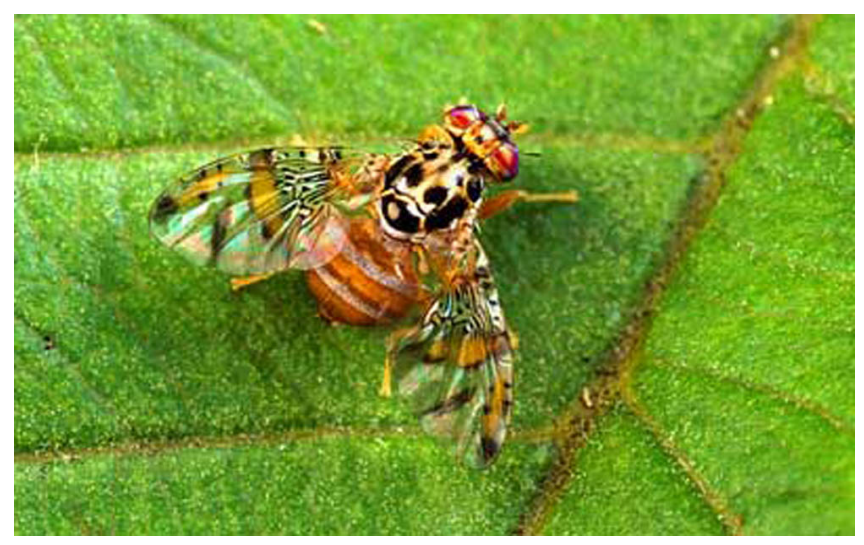

Figure 11. Dorsal view of adult male Mediterranean fruit fly, Ceratitis capitata (Wiedemann). Credits: Scott Bauer, USDA 


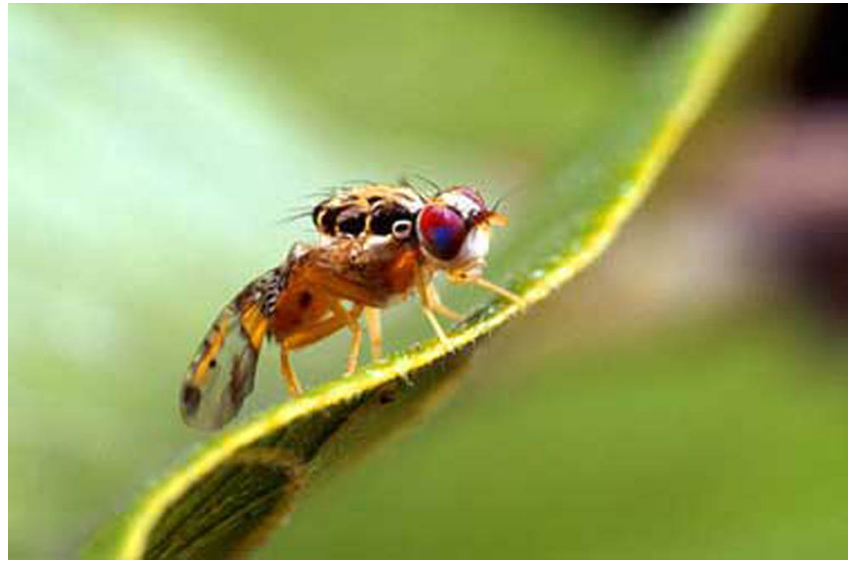

Figure 12. Lateral view of adult Mediterranean fruit fly, Ceratitis capitata (Wiedemann). Credits: Scott Bauer, USDA

\section{Life History and Habits}

The length of time required for the medfly to complete its life cycle under typical Florida summer weather conditions, and on which eradication schedules in Florida are based, is 21 to 30 days. A female medfly will lay one to 10 eggs in an egg cavity $1 \mathrm{~mm}$ deep, may lay as many as 22 eggs per day, and may lay as many as 800 eggs during her lifetime (usually about 300). The number of eggs found at any time in the reproductive organs is no indication of the total number of eggs an individual female is capable of depositing, as new eggs are being formed continually throughout her adult life. Females usually die soon after they cease to oviposit.

Eggs are deposited under the skin of fruit which is just beginning to ripen, often in an area where some break in the skin already has occurred. Several females may use the same deposition hole with 75 or more eggs clustered in one spot. When the eggs hatch, the larvae promptly begin eating, and at first tunnels are formed, but may keep close together in feeding until nearly full grown. Fruit in a hard or semiripe condition is better for oviposition than fully ripened fruit. Ripe fruit is likely to be more juicy, and such fruits often are associated with a high mortality of eggs and young larvae.

Females will not oviposit when temperatures drop below $60.8^{\circ} \mathrm{F}\left(16^{\circ} \mathrm{C}\right)$ except when exposed to sunlight for several hours. Development in egg, larval, and pupal stages stops at $50^{\circ} \mathrm{F}\left(10^{\circ} \mathrm{C}\right)$. Pupae carry the species through unfavorable conditions, such as lack of food, water, and temperature extremes. During warm weather eggs hatch in 1.5 to three days. The duration of the egg stage is considerably increased by lower temperatures.

Larvae pass through three instars. Larval life may be as short as six to 10 days when the mean temperatures average $77-79^{\circ} \mathrm{F}\left(25-26.1^{\circ} \mathrm{C}\right)$. The kind and condition of the fruit often influence the length of the larval stage. In citrus fruits, especially limes and lemons, it appears to be longer. Thus larvae require 14 to 26 days to reach maturity in a ripe lemon, as compared with 10 to 15 days in a green peach. Larvae leave the fruit in largest numbers at or just after daybreak and pupate in the soil or whatever is available.

Minimum duration of the pupal stage is six to 13 days when the mean temperature ranges from about $76-79^{\circ} \mathrm{F}\left(24.4-26.1^{\circ} \mathrm{C}\right)$. Back and Pemberton (1915) noted that this period may be increased to at least 19 days when the daily temperature means drop to about $69-71^{\circ} \mathrm{F}\left(20.6-21.7^{\circ} \mathrm{C}\right)$.

Adults emerge in largest numbers early in the morning during warm weather and emerge more sporadically during cool weather. They can fly short distances, but winds may carry them a mile or more away. Copulation may occur at any time throughout the day. Newly emerged adults are not sexually mature. Males often show sexual activity four days after emergence, and copulation has been observed five days after emergence. Both sexes are sexually active throughout the day. When the daily mean temperature averages from $76-78^{\circ} \mathrm{F}(24.4$ $25.6^{\circ} \mathrm{C}$ ), most females are ready to mate from six to eight days after eclosion. Oviposition may take place as early as four to five days after emergence during very warm weather, but not for about 10 days when temperatures range between $68-72^{\circ} \mathrm{F}(20$ 22. ${ }^{\circ} \mathrm{C}$ ) (Back and Pemberton 1915).

Adults die within four days if they cannot obtain food. Usually about $50 \%$ of the flies die during the first two months after emergence. Some adults may survive up to six months or more under favorable conditions of food (fruit, honeydew, or plant sap), water, and cool temperatures. When host fruit is continuously available and weather conditions 
favorable for many months, successive generations will be large and continuous. Lack of fruit for three to four months reduces the population to a minimum.

\section{Hosts}

The Mediterranean fruit fly attacks more than 260 different fruits, flowers, vegetables, and nuts. Thin-skinned, ripe succulent fruits are preferred. Host preferences vary in different regions. Although several species of cucurbits have been recorded as hosts of the medfly, they are considered to be very poor hosts. Some hosts have been recorded as medfly hosts only under laboratory conditions and may not be attacked in the field. Knowledge of the hosts in one country often aids in correctly predicting those which are most likely to be infested in a newly infested country, but what may be a preferred host in one part of the world may be a poor host in another.

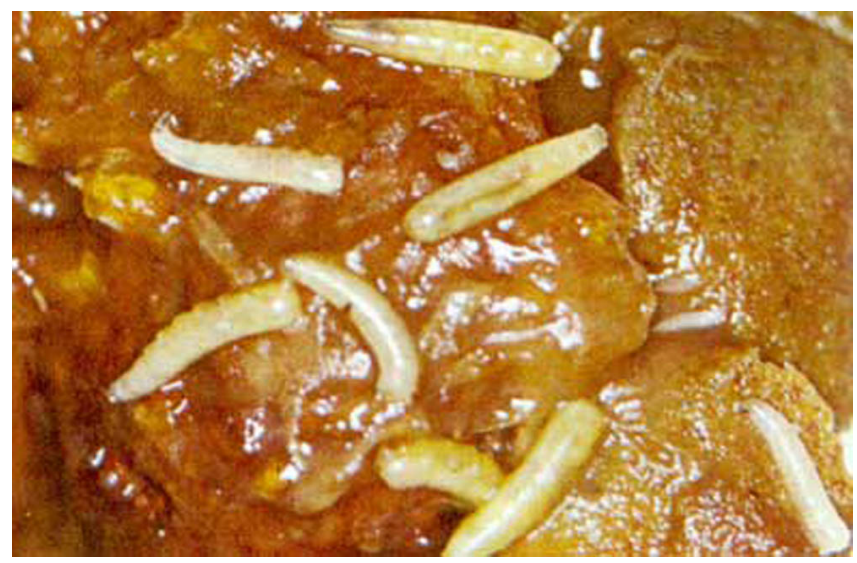

Figure 14. Peach infested with larvae of the Mediterranean fruit fly, Ceratitis capitata (Wiedemann). Credits: USDA

\section{Mediterranean Fruit Fly Host List}

Attached is a world list of hosts grouped according to their importance according to best available information.

\section{Heavily or Generally Infested}

- Blighia sapida K König (Cupania sapida (K König) Voigt), akee.

- Calophyllum inophyllum L., indiapoon beauty-leaf, kamani, Alexander laurel.

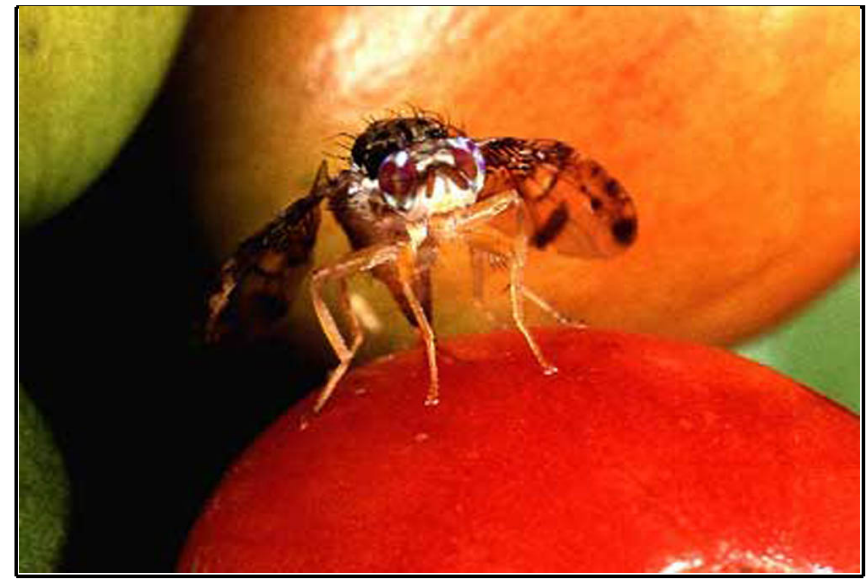

Figure 15. A female Mediterranean fruit fly, Ceratitis capitata (Wiedemann), pumps eggs through her ovipositor into the soft outer layers of a ripe coffee berry. Credits: Scott Bauer, USDA

- Casimiroa edulis Llave, white sapote, casimiroa, Mexican apple.

- Chrysophyllum cainito L., cainito, star apple.

- Chrysophyllum oliviforme L., satin leaf, star apple, caimitillo, damson plum (Jamaica).

- Chrysophyllum polynesicum Hillebr., chrysophyllum.

-X Citrofortunella mitis (Blanco) J. Ingram and H.E. Moore (Citrus mitis Blanco), calamondin, Panama orange.

- Citrus aurantiifolia (Christm.) Swingle, lime.

- Citrus aurantium L., sour orange, Seville orange, bitter-sweet orange; (C. myrtifolia Raf.), myrtle-leaf orange.

- Citrus limon (L.) Burm. f., lemon, except 'Eureka', 'Lisbon', and 'Villa Franca' cultivars (smooth-skinned sour lemon).

- Citrus x limonia Osbeck (C. taitensis Risso), lemon.

- Citrus maxima (Burm.) Merrill (C. grandis (L.) Osbeck; C. decumana (L.) L.), pummelo, pomelmous shaddock, Pernambuco.

- Citrus x nobilis Lour., king orange. 
- Citrus x paradisi Macfady, grapefruit, pomelo.

- Citrus reticulata Blanco (C. deliciosa Ten.; $C$. nobilis Andr. var. deliciosa Ten.), mandarin orange, tangerine.

- Citrus sinensis (L.) Osbeck 'Valencia' and 'Parson Brown' and 'Lue Gim Gong', Malta orange, Lambs summer orange.

- Coffea canephora Pierre ex Froehn., robusta coffee, Coffea arabica L., Arabian coffee, common coffee.

- Coffea liberica Bull ex Hiern, Liberian coffee.

- Cydonia oblonga Mill. (C vulgaris Pers.), quince, mannela.

- Diospyros decandra Lour., persimmon.

- Diospyros kaki L. f. (D. chinensis Blume; D. roxburghii Carr.; D. schi-tse Bunge), kaki persimmon, Oriental persimmon.

- Dovyalis caffra (Hook. f. \& Harv.) Warb. (Aberia caffra Hook. f. \& Harv.), kei-apple, umkokolo.

- Eriobotrya japonica (Thunb.) Lindl. (Photinia japonica Thunb.), loquat, Malta plum.

- Eugenia uniflora L. (E. michelii Lam.; Stenocalyx michelii Berg), Surinam cherry, pitanga, Brazil cherry, cayenne cherry, Florida cherry, French cherry.

- Ficus carica L., common fig, lemon fig.

- Fortunella japonica (Thunb.) Swingle (Citrus japonica Thunb.), round and marumi kumquat.

- Malus pumila Mill. (M. communis Poir.; Pyrus pumila (Mill.) C. Koch), common apple.

- Mangifera indica L., mango.

- Mimusops elengi L., elengi tree, pogada, West Indian medlar, elengi bulletwood.

- Murraya paniculata (L.) Jacq. (M. exotica L.), orange-jessamine, mock orange.
- Prunus americana Marsh., native plum, American plum.

- Prunus armeniaca L. (Armeniaca vulgaris Lam.), apricot.

- Prunus domestica L., garden plum, Prunus domestica L. spp. insitita (L.) Schneid., damson,bullace.

- Prunus persica (L.) Batsch. (Amygdalus persica L.; Mill.), Persica vulgaris (Mill.), peach.

- Prunus persica (L.) Batsch. var. nucipersica (Suckow) C.K. Schneid. (P. persica (L.) Batsch. var. nectarina (Ait. f.) Maxim.), nectarine.

- Psidium cattleianum Sab. (P. littorale Raddi var. longipes (O. Berg.) Fosb.), strawberry guava, cattley guava, waiawi.

- Psidium guajava L., guava.

- Pyrus communis L., common pear.

- Pyrus x leconte Rehd., leconte pear.

- Syzygium jambos (L.) Alston (Eugenia jambos L.; Caryophyllus jambos Stokes), rose apple, jambos, Malabar plum.

- Terminalia catappa L., tropical almond, false kamani, winged kamani.

- Terminalia chebula Retz., black myrobalan, chebula terminalia.

\section{Occasionally Infested}

- Annona muricata L., soursop, guan -bana.

- Averrhoa carambola L., carambola, star-fruit.

- Capsicum annuum L. (C. frutescens auct.; $C$. baccatum Vell.), Conoides Group: red pepper; Grossum Group: bell pepper, sweet pepper; Cerasiforme Group: cherry pepper; Longum Group: cayenne pepper, chili, long red.

- Carica papaya L., papaya, papaw. 
- Carica quercifolia Solms, dwarf papaya, oakleaf papaya.

- Carissa bispinosa (L.) Desf. ex Brenan (C. arduina Lam.; C. acuminata A. DC.), hedge thorn.

- Fragaria x ananassa Duchesne (Fragaria chilonensis (L.) Duchesne $\mathrm{x} F$. virginiana Duchesne), cultivated or garden strawberry.

- Garcinia mangostana L., mangosteen.

- Garcinia xanthochymus Hook. f. ex T. Anderson, garcinia, gourka.

- Gossypium sp., cotton.

- Juglans sp., walnut.

- Lycopersicon esculentum Mill., tomato.

- Ochrosia elliptica Labill., ochrosia.

- Opuntia sp., prickly pear. O. humifusa (Raf.) Raf. (O. compressa (Salisb.) Macbr.; O. opuntia (L.) Karst.; $O$. rafinesquei Engelm.; $O$. mesacantha Rafin.), O. humifusa Raf. 'Variegata', O. dilleni Haw., O. tuna (L.) Mill.(O. humilis Haw.; O. horrida Salm-Dyck ex DC.), O. polyantha Haw., O. vulgaris Mill. (O. monocantha (Willd.) Haw.; O. nana DC.).

- Opuntia ficus-indica (L.) Mill. (O. engelmanni Salm-Dyck; $O$. megacantha Salm-Dyck; $O$. occidentalis Engelm. \& Bigel.), Indian fig, spineless cactus.

- Persea americana Mill. (P. gratissima C.F. Gaertn.), avocado, alligator pear.

- Syzygium malaccense (L.) Merrill \& L.M. Perry (Eugenia malaccensis L.; Jambosa malaccensis (L.) DC.; Caryophyllus malaccensis Stokes), ohia, Malay apple, pomerack, mountain apple.

\section{$\underline{\text { Rarely Infested }}$}

- Annona reticulata L., bullocks-heart, custard apple, anona.

- Arenga pinnata (Wurmb) Merrill, (A. saccharifera Labill.), gomuti, sugar palm.
- Artocarpus altilis (Parkins.) Fosb. (A. incisus L.F.; A. communis Forst.), breadfruit.

- Carissa grandiflora (E.H. Mey.) A. DC., Natal plum, carissa.

- Cestrum sp., cestrum, jessamine: C. nocturnum L., night-jessamine, Chinese inkberry, night-blooming jasmine.

- Clausena lansium (Lour.) Skeels (C. punctata (Sonn.) Rehd. \& E.H. Wils.; C. wampi (Blanco) D. Oliver), Chinese wampee, wampi.

- Latania loddigesii Mart. (L. glaucophylla Hort. ex Baker), blue palm.

- Litchi chinensis Sonn. (Nephelium litchi Camb.), litchi, lychee.

- Lycium europaeum L., European wolfberry.

- Malpighia glabra L., Barbados cherry, acerola.

- Manilkara zapota (L.) Van Royen (Achras zapota L.; Sapota achras Mill.; M. zapotilla (Jacq.) Gilly), sapodilla.

- Musa acuminata Colla (M. cavendishii Lamb. ex Paxt.; M. nana auct.; M. chinensis Sweet), dwarf banana, Chinese banana.

- Musa x paradisiaca L. (M. x sapientum L.), common banana, plantain.

- Noronhia emarginata (Lam.) Thouars ex Hook., Madagascar olive, noronhia, Chinese plum.

- Passiflora sp., passion flower, P. caerulea L., blue-crown passion flower, $P$. foetida $\mathrm{L}$., tagua passion flower.

- Phoenix dactylifera L., date palm.

- Punica granatum L., pomegranate.

- Rubus sp., blackberry, youngberry.

- Santalum freycinetianum Gaudich. (S. paniculatum Hook. \& Arn.), beach sandalwood. 
- Solanum incanum L. (S. coagulans Forssk.).

- Solanum melongena L. var. esculentum Nees, garden eggplant.

- Spondias cytherea Sonn. (S. dulcis G. Forst.), ambarella, otaheite apple, vi-apple, wi.

- Syagrus campestris (Mart.) H. Wendl. (Cocos campestris Mart.), field syagrus palm.

- Syzygium samarangense (Blume) Merrill \& L.M. Perry (Eugenia javanica Lam.).

- Vitis lambrusca L., fox grape, Isabella grape.

\section{Laboratory Infestations}

- Acanthocereus sp., acanthocereus. A. pentagonus (L.) Britt. \& Rose (Cereus baxaniensis Karw. ex Pfeiff.; C. pentagonus (L.) Haw.), barbwire acanthocereus, dildoe, cactus.

- Aronia arbutifolia (L.) Pers. (Pyrus arbutifolia (L.) L.f.), red chokeberry.

- Coccoloba diversifolia Jacq. (C. floridana Meissn.; C. laurifolia Lindau), pigeon plum, dove plum.

- Crataegus floridana Sarg., Jacksonville hawthorn.

- Crataegus galbana Beadle, hawthorn.

- Cucumis anguria L. (C. erinaceus Hort.; $C$. grossulariiformis Hort.), West Indian gherkin, wild cucumber.

- Cydonia sp., quince. Cydonia sinensis Thouin (Chaenomeles sinensis (Thouin) Koehne; Pseudocydonia sinensis Schneid.), Chinese quince.

- Echinocereus triglochidiatus Engelm. var. neomexicanus (Standl.) Standl. ex W.T. Marsh. (E. polyacanthus Engelm.), cactus.

- Eugenia axillaris (Swartz) Willd., whitestopper eugenia.

- Forestiera sp. (Adelia sp.), adelia. F. segregata (Jacq.) Krug \& Urban, Florida privet.
- Fortunella hindsii (Chapm.) Swingle, Hong Kong kumquat.

- Fragaria chiloensis (L.) Duchesne, strawberry, chiloe strawberry.

- Hibiscus sabdariffa L., roselle, Jamaica sorrell.

- Hylocereus undatus (Haw.) Britt. \& Rose (Cereus undatus Haw.), night-blooming cereus, pitaya.

- Ilex glabra (L.) A. Gray, inkberry, gallberry.

- Licania michauxii Prance (Geobalanus oblongifolius Michx.), gopher apple.

- Osmanthus americanus (L.) A. Gray (O. floridanus Chapm.), wild olive, hammock osmanthus.

- Solanum erianthum D. Don (S. verbascifolium auct.), potato tree, mullein nightshade.

- Solanum seaforthianum Andr., Brazilian nightshade.

- Solanum sisymbriifolium Lam., nightshade.

- Ximenia americana L., tallow-wood.

\section{Unknown Importance}

- Acokanthera sp. (Toxicophlaea). A. longiflora Stapf, Bushman's-poison.

- Ananas comosus (L.) Merrill (A. sativus Schult.), pineapple.

- Annona cherimola Mill., cherimoya.

- Annona glabra L., pondapple, alligator apple.

- Annona squamosa L., sugar apple, sweetsop.

- Arbutus unedo L., strawberry madrone.

- Argania spinosa (L.) Skells (A. sideroxylon Roem. \& Schult.), hardwood evergreen tree, Morocco ironwood.

- Argemone mexicana L., Mexican prickle poppy, cardosanta, cardo. 
- Artabotrys hexapetalus (L.f.) Bhand. (A. uncinatus (Lam.) Merrill; A. odoratissimus R. $\mathrm{Br}$.), fragrant tailgrape, climbing ylang-ylang.

- Asimina triloba (L.) Dunal, papaw, pawpaw.

- Asimina obovata (Willd.) Nash, bigflower pawpaw.

- Asimina parviflora (Michx.) Dunal, smallflower pawpaw.

- Asimina pygmaea (Bartr.) Dunal, sprawling pawpaw.

- Asimina reticulata Schuttlw. ex Chapm., seminoletea pawpaw, common pawpaw.

- Asparagus densiflorus (Kunth) Jessop 'Sprengeri', Sprenger asparagus.

- Atropa belladonna L., belladonna.

- Berberis holstii Engl., barberry.

- Brucea ferruginea L'Her. (B. antidysenterica Lam.).

- Bumelia lycioides (L.) Pers., buckthorn bumelia.

- Bumelia tenax (L.) Willd., tough bumelia, buckthorn.

- Butia sp., butia palm. B. capitata (Mart.) Becc. (Cocos capitata Mart.), jelly palm, Brazilian butia palm, pindo palm.

- Cananga odorata (Lam.) Hook. f. \& T. Thoms., ylang-ylang.

- Capparis citrifolia Lam., caper.

- Carissa carandas L., karanda carissa.

- Chrysobalanus ellipticus Soland. ex Sabine.

- Chrysobalanus icaco L., icaco coco plum, gopher apple, gopher plum.

- Chrysophyllum africanum A. DC. (C. argyrophyllum Hiern), African star apple.
- Chrysophyllum viridifolium Wood \& Franks.

- Citharexylum fruticosum L. (C. cinereum L.), Florida fiddlewood.

- Citrullus lanatus (Thunb.) Matsum. \& Nakai (C. vulgaris Schrad.), watermelon.

- Citrus medica L., citron.

- Clintonia umbellulata (Michx.) Morong, spreckled beadlily.

- Coccoloba uvifera (L.) L., sea grape.

- Cotoneaster adpressus Bois. var. praecox Bois. \& Berthault (C. praecox (Bois \& Berthault) Hort. Vilm. - Andr. ex Meuniss.), early creeping cotoneaster.

- Crataegus azarolus L., azarole hawthorn, haw.

- Crinum asiaticum L. (C. sinicum Roxb. ex Herb.) St. John's lily.

- Cucumis dipsaceus C.G. Ehrenb. ex Spach, hedgehog, teaselgourd, wild cucumber.

- Cucumis melo L., Cantalupensis Group: cantaloupe; Inodorns Group: casaba melon; Reticulatus Group: muskmelon.

- Cucumis sativus L., cucumber.

- Cucurbita maxima Duchesne, winter squash, hubbard squash.

- Cucurbita moschata (Duchesne) Poir., cushaw pumpkin, Canada and winter crookneck pumpkin.

- Cucurbita pepo L., pumpkin, vegetable marrow.

- Cyphomandra betacea (Cav.) Sendtn., tree tomato.

- Diospyros mespiliformis Hochst. ex A. DC., medlar persimmon.

- Diospyros virginiana L., common persimmon, wild persimmon. 
- Dovyalis hebecarpa (G. Gardn.) Warb., kitembilla, Ceylon gooseberry.

- Ekebergia capensis Sparrm., dog plum.

- Eugenia brasiliensis Lam. (Stenocalyx brasiliensis Berg), Brazil eugenia, Brazilian plum, Spanish cherry.

- Euphorbia lathyris L., caper euphorbia, gopher apple.

- Euphoria longan (Lour.) Steud. (Nephelium longan Lour.; Dimocarpus longan Lour.), longan, dragon's eye.

- Feijoa sellowiana O. Berg, feijoa guavasteen, pineapple guava.

- Ficus benghalensis L. (F. indica L.), glabrous tree, India fig.

- Flacourtia indica (Burm. f.) Merrill (F. ramontchi L'Her.), ramontchi, governor's plum.

- Fortunella crassifolia Swingle, meiwa kumquat.

- Fortunella margarita (Lour.) Swingle, Eustis limequat, megami kumquat.

- Gardenia sp., gardenia.

- Glycosmis pentaphylla (Retz.) Correa, glycomis, Malay glycosmis.

- Harpephyllum caffrum Bernh. ex C.F. Krauss, Kafir plum.

- Hevea brasiliensis (Willd. ex A. Juss.) Mull. Arg., para rubber tree, caoutchoue, Brazil rubber.

- Homalocladium platycladum (F.J. Muell.) L.H. Bailey, ribbon bush.

- Ilex vomitoria Ait., Carolina holly, yaupon.

- Juglans hindsii (Jeps.) Jeps.

- Landolphia sp., gumvine.

- Lycium carolinanum Walt., Carolina wolfberry, boxthorn.
- Lycium chinense Mill. (L. carnosum Hort.), (L. campanulatum Drege ex Dun.), boxthorn.

- Lycium horridum Thunb., African buckthorn.

- Maclura pomifera (Raf.) C.K. Schneid. (M. aurantiaca Nutt.; Toxylon pomifera Raf.), osage orange.

- Malpighia punicifolia L., Barbados cherry, acerola.

- Mammea americana L., mamey, mammee apple.

- Marrubium vulgare L., common hoarhound.

- Mastichodendron foetidissimum (Jacq.) Lam. (Sideroxylon mastichodendron Jacq.; Sideroxylon foetidissimum Jacq.), mastic, jungle plum, ironwood.

- Melicoccus bijugatus Jacq. (Melicocca bijuga L.), mamoncillo, mamon, Spanish lime, genip.

- Melothria pendula L., creeping cucumber.

- Mespilus germanica L., medlar.

- Mimusops sp., milkwood.

- Mimusops caffra E.H. Mey. ex A. DC., Kafir bulletwood.

- Mimusops kirkii Bak.

- Momordica balsamina L., balsam apple.

- Monstera deliciosa Liebm. (Philodendron pertusum Kunth \& Bouche), ceriman.

- Morus sp., mulberry.

- Myrciaria edulis (Vell.) Skeels (Eugenia edulis Vell.), willow-leaved eugenia.

- Nyssa ogeche Bartr., ex Marsh., ogeche lime, ogechi plum, ogeechee tupelo.

- Nyssa sylvatica Marsh., sour gum.

- Nyssa sylvatica Marsh. var. biflora (Walt.) Sarg., black gum, swamp black tupelo. 
- Olea europaea L., common olive.

- Passiflora edulis Sims, purple granadilla, lilikoi, passion fruit.

- Passiflora incarnata L., wild passion flower, maypop.

- Passiflora laurifolia L., yellow granadilla, water lemon, Jamaica honeysuckle.

- Passiflora lingularis Juss., sweet granadilla.

- Passiflora mollissima (HBK) L.H.Bailey, lilikoi, soft-leaf passion flower.

- Passiflora quadrangularis L. (P. macrocarpa M.T. Mast.), giant granadilla.

- Peponia mackennii Naud., wild cucurbit.

- Pereskia aculeata Mill. (P. pereskia (L.) Karst.), Barbados gooseberry.

- Phaseolus limensis Macfady., lima bean.

-Phaseolus lunatus L., sieva bean, cibet bean.

- Phaseolus vulgaris L., kidney bean, haricot, string bean.

- Phyllantus acidus (L.) Skeels, otaheite gooseberry, leafflower, Ceylon gooseberry.

- Physalis peruviana L., cape gooseberry, poha.

- Pimenta dioica (L.) Merrill (P. officinalis Lindl.), allspice, pimenta.

- Pleiogynium cerasiferum (F.J. Muell.) R. Parker ( $P$. solandri (Benth.) Engl.; Spondias solandri Benth.), burdekin plum.

- Podocarpus elongatus (Ait.) L'Her. ex Pers., yellowood, fern podocarpus.

- Poncirus trifoliata (L.) Raf., trifoliate orange.

- Pouteria campechiana (HBK) Baehni (Lucuma nervosa A. DC.; L. rivicoa Gaertn. f.), canistel incuma, egg fruit.

- Pouteria sapota (Jacq.) H.E. Moore \& Stern (Calocarpum sapota (Jacq.) Merrill; $C$. mammosum auct.; Lucuma mammosa auct.), sapote.

- Prunus cerasus L. (Cerasus caproniana DC.), sour cherry.

- Prunus dulcis (Mill.) D.A. Webb (P. amygdalus Batsch; P. communis (L.) Arcang.) almond.

- Prunus japonica Thunb., Chinese bushcherry, plum.

- Prunus salicina Lindl., Japanese plum.

- Prunus umbellata Ell., wild plum, flatwoods plum.

- Psidium guineense Swartz, Brazilian guava.

- Psidium littorale Raddi var. littorale (P. littorale Raddi var. lucidum (Degener) Fosb.), yellow cattley guava.

- Putranjiva roxburghii Wallich, wild olive, India amulet plant.

- Pyracantha coccinea M.J. Roem. 'Lalandei', laland firethorn.

- Ribes sp., currant, gooseberry.

- Robinia sp., locust.

- Rosa sp., rose.

- Royena pallens Thunb. (R. pubescens Willd.), pale-branched royena.

- Salix sp., willow leaf.

- Santalum album L., sandalwood, white sandalwood.

- Scaevola plumieri (L.) Vahl, goodenia beechberry.

- Schinus molle L., California pepper tree (blossoms).

- Sechium edule (Jacq.) Swartz (Chayota edulis Jacq.); chayote, christophine. 
- Selenicereus pteranthus (Link \& Otto) Britt. \& Rose (Cereus nycticalus Link; Cereus pteranthus Link ex Dietr.), cactus.

- Serenoa repens (Bartr.) Small (S. serrulata (Michx.) Nichols; Brahea serrulata H. Wendl.; Chamerops serrulata Michx.; Sabal serrulatum Shult. f.), saw palmetto.

- Severinia buxifolia (Poir.) Ten. (Atalantia buxifolia (Poir.) D. Oliver; Triphasia monophylla DC.), Chinese box orange.

- Sideroxylon inerme L., ironwood.

- Smilax beyrichii Kunth, Smilax sandwicensis Kunth, smilax.

- Solanum aculeatissimum Jacq., solanum, Sodoms apple, nightshade.

- Solanum capsicastrum Link ex Schauer, solanum cherry, false Jerusalem cherry.

- Solanum carolinense L., Caroline horse nettle.

- Solanum melanocerasum All. (S. nigrum L. var. guineense L.), solanberry, black nightshade, garden huckleberry.

- Solanum pseudocapsicum L., Jerusalem cherry.

- Solanum sodomeum L., apple of Sodom.

- Sorbus sp., mountain ash.

- Spondias sp., West Indian plum.

- Spondias mombin L. (S. axillaris Roxb.; S. lutea L.), yellow mombin, Spanish plum, hog plum, jobo, cajamerin.

- Strychnos atherstonei Harv., cape teak.

- Strychnos pungens Solered., poison nut, wild orange.

- Syzygium cumini (L.) Skeels (S. jambolana (Lam.) DC. Eugenia cumini (L.) Druce; E. jambolana Lam.), jambolan, Java palm.

- Teclea trichocarpa (Engl.) Engl.
- Terminalia pallida Brandis, terminalia.

- Theobroma cacao L., cacao, cocoa.

- Thevetia peruviana (Pers.) K. Schum. (T. neriifolia A. Juss. ex Steud.; Cerbera thevetia L.), yellow oleander, be-still, lucky nut thevetia.

- Vaccinium cereum Forst. f., blueberry.

- Vangueria edulis Vahl (V. madagascariensis J.F. Gmel.), vangueria.

- Vangueria infausta Burchell, wild medlar, vangueria.

- Vicia faba L., broad bean, horse bean.

- Vitis sp. (hybrid), beacon grape.

- Vitis vinifera L., wine grape, European grape.

- Wikstroemia phillyreifolia Gray, wikstroemia.

-Ziziphus jujuba Mill. (Z. vulgaris Lam., Z. sativa Gaertn.), jujube, Chinese date.

\section{Detection and Survey}

A primary method of collecting larvae is by cutting infested fruit. Fully grown larvae, when the surrounding air temperature is warm, flex and "jump" repeatedly as much as $25 \mathrm{~mm}$ when removed from fruit. Adults are collected primarily by use of sticky-board traps and baited traps. Larval identification is extremely difficult, so that when feasible it is best to rear them to adults for identification. If collected larvae must be killed, they should be placed in hot water and then transferred to $70 \%$ isopropenol. Larval identification is based primarily on characters of mature 3rd instar larvae.

\section{Management}

As a Mediterranean fruit fly infestation falls under the control of Florida's regulatory agency - the Department of Agriculture and Consumer Services and its Division of Plant Industry, there are no University of Florida management recommendations. Plus treatment strategies are changing in an environment of public concern about aerial application of insecticidal baits. The treatment 


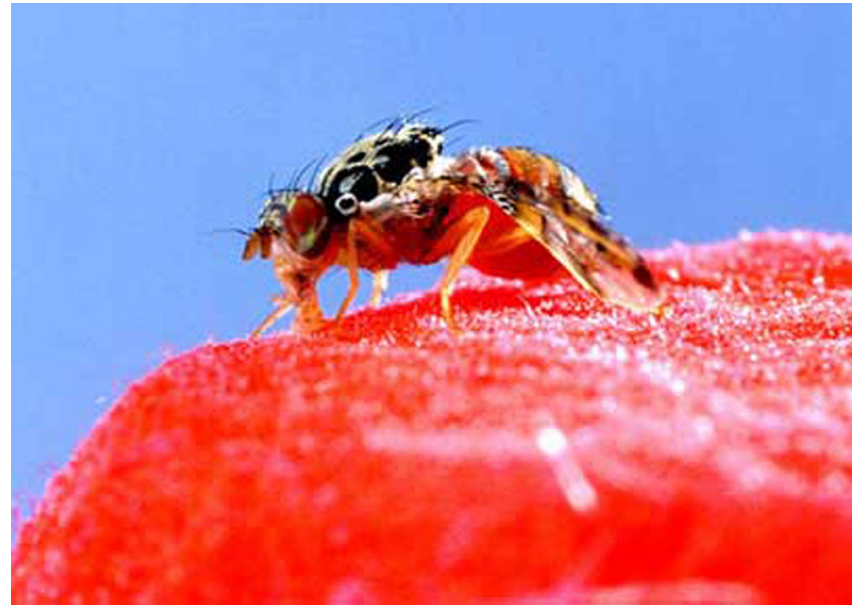

Figure 16. Adult Mediterranean fruit fly, Ceratitis capitata (Wiedemann), feeding on a cotton wick soaked with a bait-dye mixture. Credits: Scott Bauer, USDA

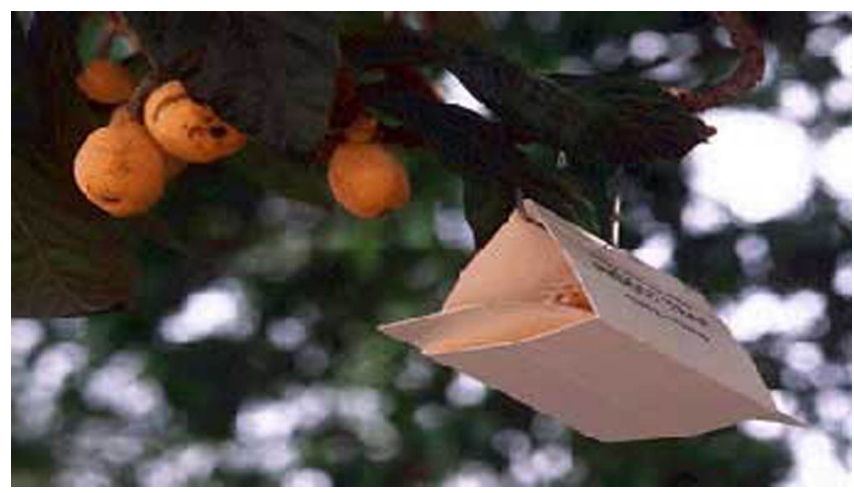

Figure 17. Trap used to capture adults of the Adult Mediterranean fruit fly, Ceratitis capitata (Wiedemann). Credits: USDA

strategies are outlined for the Miami Spring and Umatilla infestations of 1998 at Completed Medfly Programs in Florida, 1998 (Dixon and Hamon 1998), and are described in more detailed, as they occurred, in the Mediterranean Fruit Fly Pest Alert Archives (Fuller and Fasulo 1998).

ARS scientists in Hawaii and Texas are collaborating to investigate phloxine $\mathrm{B}$, better known as the FDA-approved red dye number 28 . The dye might prove a safe, effective alternative to currently used malathion and spinosad insecticide baits. Medflies often share regurgitated food. This helps spread the insecticidal dye-and-bait blend through the population (Moreno 2000).

A new technique with temperature sensitive Medflies allow for the production of medflies in the laboratory by bathing medfly eggs in warm water -- a process that kills the female embryos but doesn't

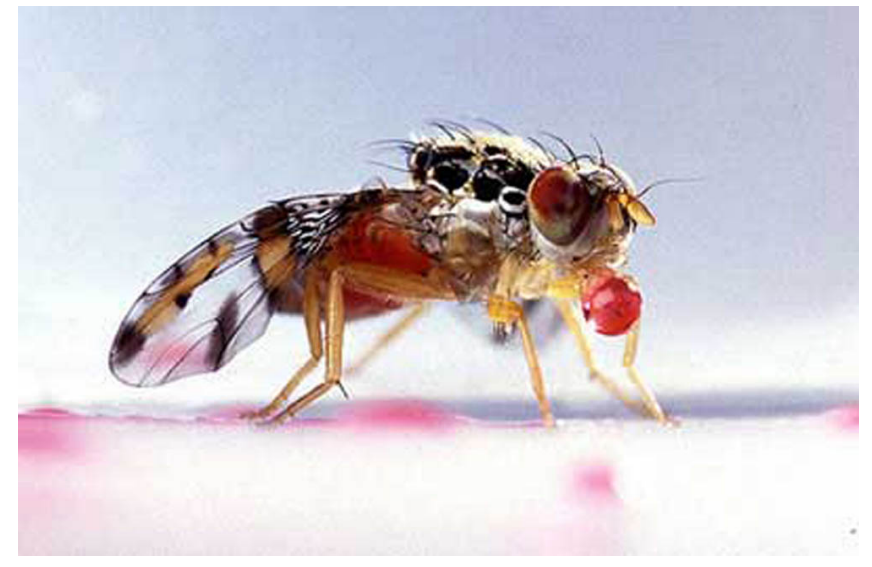

Figure 18. Lateral view of adult Mediterranean fruit fly, Ceratitis capitata (Wiedemann), regurgitating food. Credits: Scott Bauer, USDA

harm the male embryos. In the pupal stage, the males can be irradiated to render them sexually sterile (USDA 2000).

\section{Selected References}

Ayers EL. 1957. The two medfly eradication programs in Florida. Proceedings of the Florida State Horticultural Society 70: 67-69.

Back EA, Pemberton CE. 1915. Life history of the Mediterranean fruit fly from the standpoint of parasite introduction. Journal of Agricultural Research 3: 363-374. United States Department of Agriculture, Washington, D.C.

Back EA, Pemberton CE. 1918. The Mediterranean fruit fly in Hawaii. United States Department of Agriculture Bulletin 536: 1-119.

Back EA, Pemberton CE. 1918. The Mediterranean fruit fly. United States Department of Agriculture Bulletin 640: 1-43.

Berg GH. 1979. Pictorial key to fruit fly larvae of the family Tephritidae. San Salvador: Organ. Internac. Region. Sanidad. Agropee. 36 p.

Christenson LD, Foote RH. 1960. Biology of fruit flies. Annual Review of Entomology 5: 171-192.

Conley KL. 1981 (publication date not given). Field guide to medfly infestation. Cooperative Mediterranean Fruit Fly Project (California). 13 p. 
De Woskin R. 1981. Medfly training manual. Joint cooperative Mediterranean fruit fly eradication project. United States Deptartment of Agriculture, California Department of Food and Agriculture, Santa Clara County Agriculture Commissioner, Alameda County Department of Agriculture 215 p.

Dixon WN, Hamon AB. (October 1998). Completed Medfly Programs in Florida, 1998. http://www.doacs.state.fl.us/pi/enpp/ento/miamisp.html (22 June 2001).

Foote RH. 1967. Family Tephritidae. In Vanzolini, M., ed., A catalogue of the Diptera of the Americas south of the United States 57: 1-91. Dept. Zool., Sec. Agric., Sao Paulo, Brasil.

Fuller M, Fasulo TR. (1998). Mediterranean Fruit Fly Pest Alert Archives. http://extlab7.entnem.ufl.edu/PestAlert/Medfly/ medfly.htm (15 June 2001).

Greene CT. 1929. Characters of the larvae and pupae of certain fruit flies. Journal of Agricultural Research 38: 489-504.

Hardy DE. 1949. Studies in Hawaiian fruit flies. Proceedings of the Entomology Society of Washington 51: 81- 205.

Heppner JB. 1984. Larvae of fruit flies. I. Anastrepha ludens (Mexican fruit fly) and Anastrepha suspensa (Caribbean fruit fly) (Diptera: Tephritidae). Florida Department of Agriculture and Consumer Services, Division of Plant Industry Entomology Circular 260: 1-4.

Knapp JL. (1981). The Mediterranean fruit fly. EDIS http://edis.ifas.ufl.edu/CH040 (15 June 2001).

Meyer CA. 1976. Mediterranean fruit fly impact in Central America. United States Department of Agriculture Cooperative Plant Pest Report 1: 117-118.

Mitchell WC, Andrew CO, Hagen KS, Hamilton RA, Harris EJ, Maehler KL, Rhode RH. 1977. The Mediterranean fruit fly and its economic impact on Central American countries and Panama. UC/AID Pest Management and Related Environmental
Protection Project at the University of California, Berkeley. 189 p.

Moreno D. (September 2000). Field evaluation of a phototoxic dye, phloxine $\mathrm{B}$, against three species of fruit flies (Diptera: Tephritidae). USDA ARS. http://nps.ars.usda.gov/publications/ publications.htm?lognum=0000115809 (23 June 2001).

Phillips VT. 1946. The biology and identification of trypetid larvae (Diptera: Trypetidae). Memoirs of the American Entomological Society 12: 1-161.

Rhode RH, Simon J, Perdomo A, Gutierrez J, Dowling Jr. CF, Linquist DA. 1971. Application of the sterile-insect-release technique in Mediterranean fruit fly suppression. Journal of Economic Entomology 64: 708-713.

Stephenson DP. Mediterranean fruit fly not present in New Zealand. StephensonB@ maf.govt.nz. (17 July 2001).

UF/IFAS. Anonymous. (1998). Impact of Meditrrranean fruit fly on dooryard fruits. University of Florida. http://entnemdept.ifas.ufl.edu/dooryard.htm (15 June 2001).

USDA. Anonymous. (June 2000). For males only: temperature-sensitive Medflies. Agricultural Research Magazine. United States Deptartment of Agriculture, Washington, D.C. http://www.ars.usda.gov/is/AR/archive/jun00/ flies0600.htm (23 June 2001).

USDA. New Pest Detection and Survey Staff. 1975. Mediterranean fruit fly in the U.S. - 1975. Cooperative Economic Insect Report 25: 825-839. Plant Protection and Quarantine Programs, Animal and Plant Health Inspection Service, United States Department of Agriculture.

White IM, Elson-Harris MM. 1994. Fruit Flies of Economic Significance: Their Identification and Bionomics. CAB International. Oxon, UK. 601 p. 